\title{
Particle Flock Motion at Air-Water Interface Driven by Interfacial Free Energy Foraging
}

Tianqi Chen, ${ }^{a}$ Dilip K. Kondepudi, ${ }^{b}$ James A. Dixon, ${ }^{c}$ and James F. Rusling a, d, e, f

${ }^{a}$ Department of Chemistry, University of Connecticut, Storrs, CT 06269, USA.

${ }^{\mathrm{b}}$ Department of Chemistry, Wake Forest University, Winston-Salem, NC 27109, USA.

${ }^{c}$ Department of Psychology, University of Connecticut, Storrs, CT 06269, USA.

d Institute of Material Science, University of Connecticut, Storrs, CT 06269, USA.

e Department of Surgery, University of Connecticut Health Center, Farmington, CT 06032, USA.

${ }^{\mathrm{f}}$ School of Chemistry, National University of Ireland at Galway, Galway, Ireland.

Author for correspondence: James F. Rusling; e-mail: james.rusling@uconn.edu

\section{Supplementary Information}

Video S1. Flocking of 15 irregular BQ particles at the air-water interface (sped up by 2X). BQ particles first gathered into several small groups over time, then small groups merged into a large flock, and finally particles travelled around within the formed flock with constant interaction with the rest of particles outside the flock. Video S2. Gate-crossing behavior of 15 irregular BQ particles at the air-water interface (sped up by $2 \mathrm{X}$ ). The video started at 60s after adding all 15 particles into the right side of the petri dish. Flocks of BQ particles interacted with each other at the gate. At $152 \mathrm{~s}, 13$ out of $15 \mathrm{BQ}$ particles crossed the gate from right to left side.

Video S3. Video of Figure 1. Gate-crossing behavior of 15 irregular BQ particles at the air-water interface (sped up by 2X). After adding all 15 particles into the right side of the petri dish, 6 sets of 12 Samples were taken at $0 \mathrm{~s}, 10 \mathrm{~s}, 60 \mathrm{~s}, 120 \mathrm{~s}, 180 \mathrm{~s}$, and 300s (sinking) on both sides (see Table S1 for details). At 150s, 12 out of $15 \mathrm{BQ}$ particles crossed the gate from right to left side.

Video S4. Another experiment showing the gate-crossing behavior of 15 irregular BQ particles under a slightly different sampling condition (sped up by $2 \mathrm{X}$ ). After adding all 15 particles into the right side of the petri dish, 6 sets of 12 samples were taken at 0s, 10s, 60s, 120s, 202s (crossing), and 254s (sinking) on both sides. At $202 \mathrm{~s}, 12$ out of $15 \mathrm{BQ}$ particles crossed the gate from right to left side.

Video S5. Behavior of one single irregular BQ particle at the air-water interface (sped up by $2 \mathrm{X}$ ). The motion of one particle was mainly cycling (moved in a circle) and some translational movement.

Video S6. Gate-crossing behavior of one single irregular BQ particle at the air-water interface (sped up by $2 \mathrm{X})$. The single particle moved cross the gate and back again in a random manner compared to the crossing behavior as a flock shown in Video S2, S3 and S4.

\section{Experimental methods \\ Materials}

1,4-Benzoquinone (BQ), lycopodium powder and sodium chloride $(\mathrm{NaCl})$ were from Sigma Aldrich.

Nanopure water $(18 \mathrm{MO} \mathrm{cm})$ was house-distilled water purified by a Hydro Service and Supplies Picosystem (model no. ET-11218). Kapton FPC film (127 $\mu$ m thick) was from American Durafilm, rinsed with ethanol and water prior to use.

\section{Fabrication of BQ particles}

BQ powder was first molded into a pellet $(13 \mathrm{~mm}$ diameter and $1 \mathrm{~mm}$ thick) using a die and a hydraulic press (Model No. 4350L, Carver Inc.). The pellet was then broken into multiple irregular BQ particles ( 1-2 $\mathrm{mm} x-y, 1 \mathrm{~mm}$ thick). Particles shapes are mainly triangular or polygons with 3 to 5 edges. After slightly dusting with lycopodium powder, a hydrophobic material that helps particles to 'float' on the water surface and extend the observation time, the $\mathrm{BQ}$ particles were ready to use. $30 \mathrm{~mL}$ of $100 \mathrm{mM} \mathrm{NaCl}$ solution was poured into a surfactant-free glass petri dish $(90 \mathrm{~mm}$ diameter). A Kapton polymer sheet with a $3 \mathrm{~cm}$ 
rectangular opening, i.e. a gate-cut in the middle (Fig. S1, I ) was rinsed thoroughly with ethanol and water, and dried, the gate was set to sit with its bottom edge at the bottom of the dish, dividing the dish into two identical sides. Particles, initially placed on one side, can only enter the other side by crossing the gate. To begin the experiment, 15 BQ particles were introduced into the system on A side of the petri dish (Fig. S1, II ). Their motion was recorded by a high definition (HD) digital camera (GZ-E200BU, JVC) and observation was made until most particles started to sink.
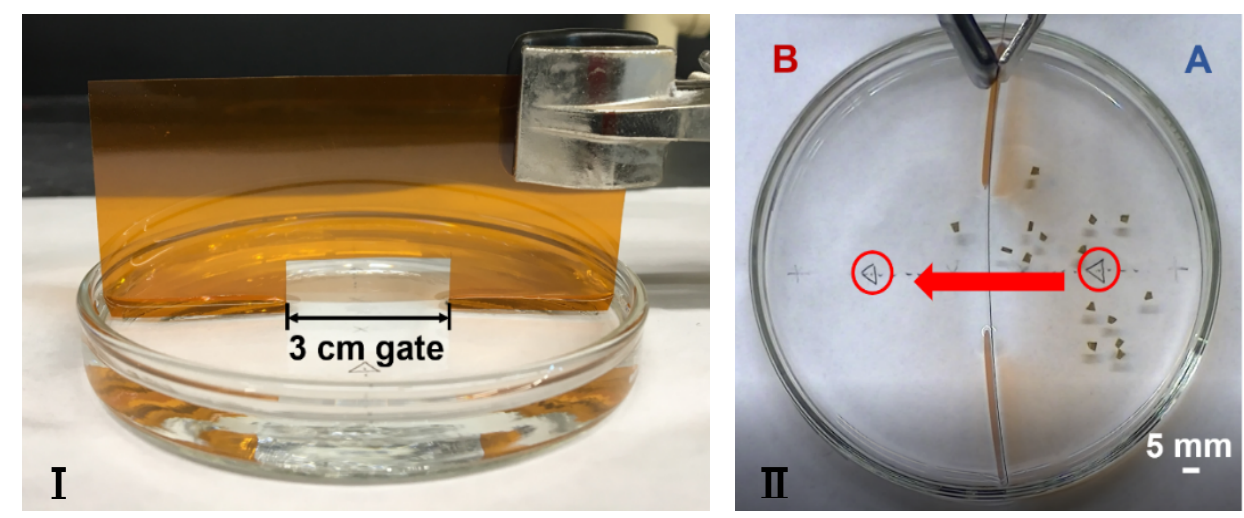

Fig S1. Two different views of the experimental set-up. ( I ) Front view. The gate was set at the bottom of the petri dish. ( II ) Top view. Particles were initially introduced into A side of the dish and observed to cross the gate travelling to B side. Samples were taken at the two triangle marks (indicated by red circles) symmetrical to the gate. Red arrow indicated the moving direction of particles.

Data collection and analysis

Surface tension and UV-visible spectra were measured on $1 \mathrm{~mL}$ samples pipetted from the petri dish at fixed times on both sides of the gate simultaneously (Fig. S1, II). Sampling parameters are listed in Table S1. Particles were observed to cross the gate at sampling times $(60 \mathrm{~s}-180 \mathrm{~s})$ (Table $\mathrm{S} 1)$.

Table S1. Sampling times of surface tension measurement

\begin{tabular}{|c|c|c|}
\hline Samples & Sampling Time(s) & Explanations \\
\hline A1 \& B1 & 0 & Blank: before adding any particle \\
\hline A2 \& B2 & 10 & 10s after adding particles \\
\hline A3 \& B3 & 60 & 1min after adding particles \\
\hline A4 \& B4 & 120 & 2min after adding particles \\
\hline A5 \& B5 & 180 & 3min after adding particles \\
\hline A6 \& B6 & 300 & Sinking moment: particles started to sink \\
\hline
\end{tabular}


Surface tension of samples was measured using EZPiplus microtensiometer (Kibron Inc.) under static (Du Noüy) surface tension mode. Surface tension vs. time curves were plotted and averaged for multiple trials under the same condition. UV absorbance of BQ was measured for samples taken at $0 \mathrm{~s}, 10 \mathrm{~s}$, crossing point and sinking point using NanoDrop 2000c Spectrophotometer (Thermo Scientific) at 245nm. The absorbance vs. time curve was generated by plotting absorbance intensity against time.
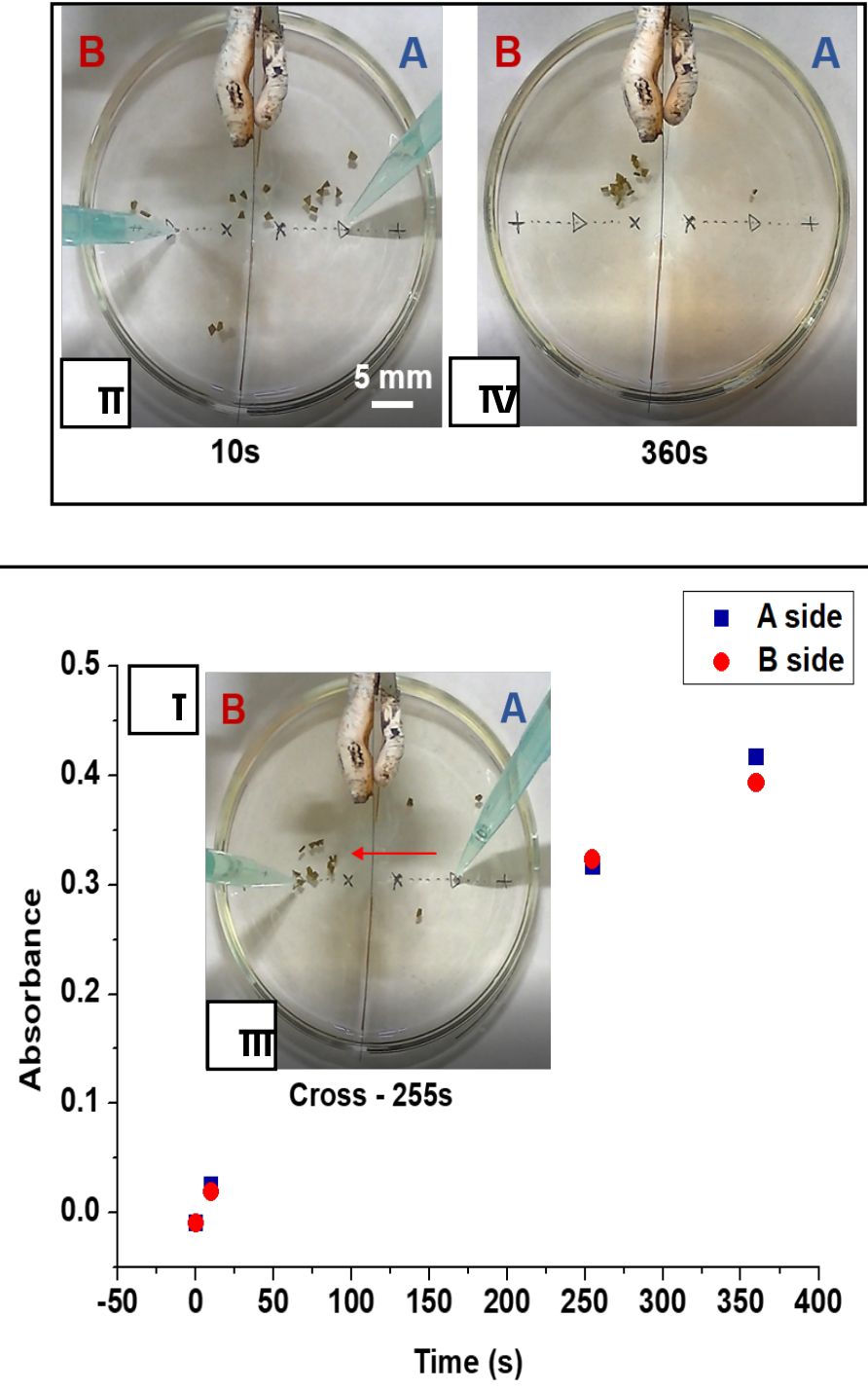

Fig S2. UV absorbance measurement and corresponding snapshots. ( I ) UV absorbance vs. time graph. 4 sets of 8 samples were taken at $0 \mathrm{~s}, 10 \mathrm{~s}, 255 \mathrm{~s}$ (crossing), and 360s (sinking) on both sides. Samples were then diluted by 50 fold and sent to UV measurement at $245 \mathrm{~nm}$. (II)-(IV) Snapshots from the video at sampling times. As shown on the graph, signals of A and B sides were close, even around the crossing point (picture II), indicating UV may not be a good method to distinguish small surface tension changes in our study. Red arrow indicated the moving direction of particles. 


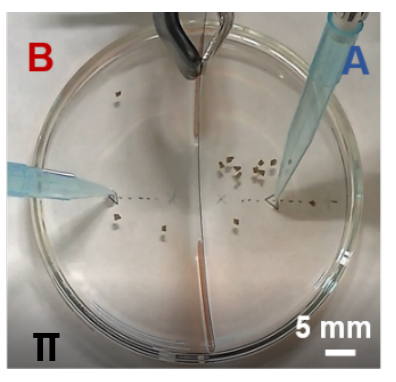

$10 \mathrm{~s}$
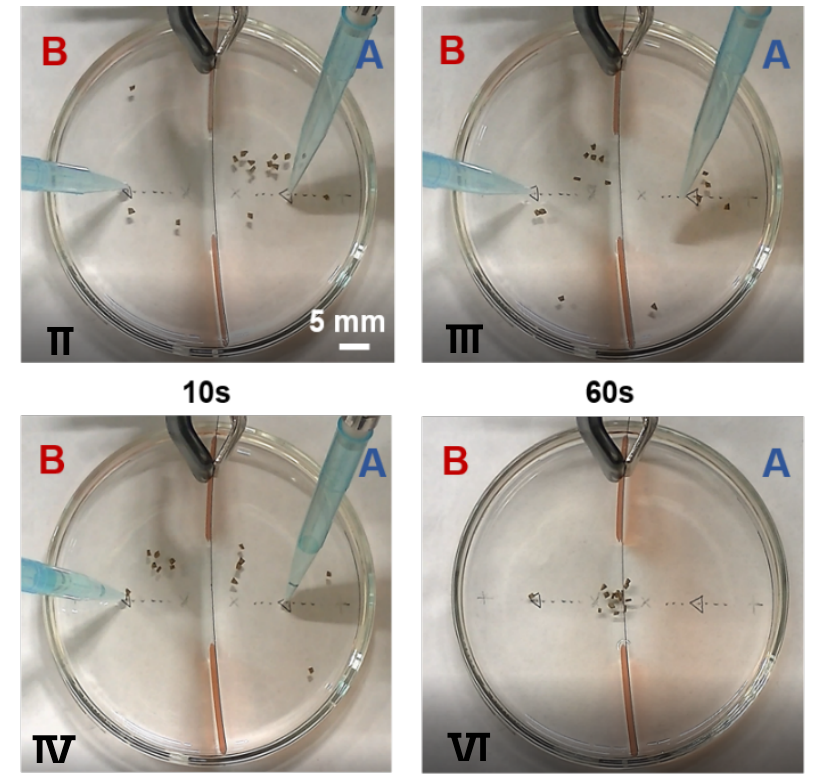

120s

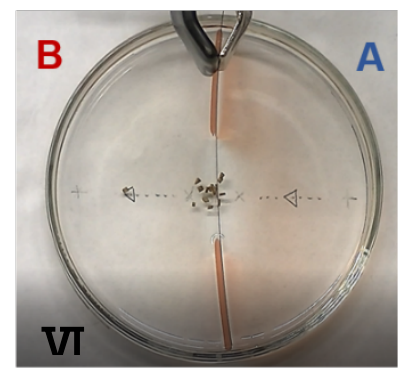

254s

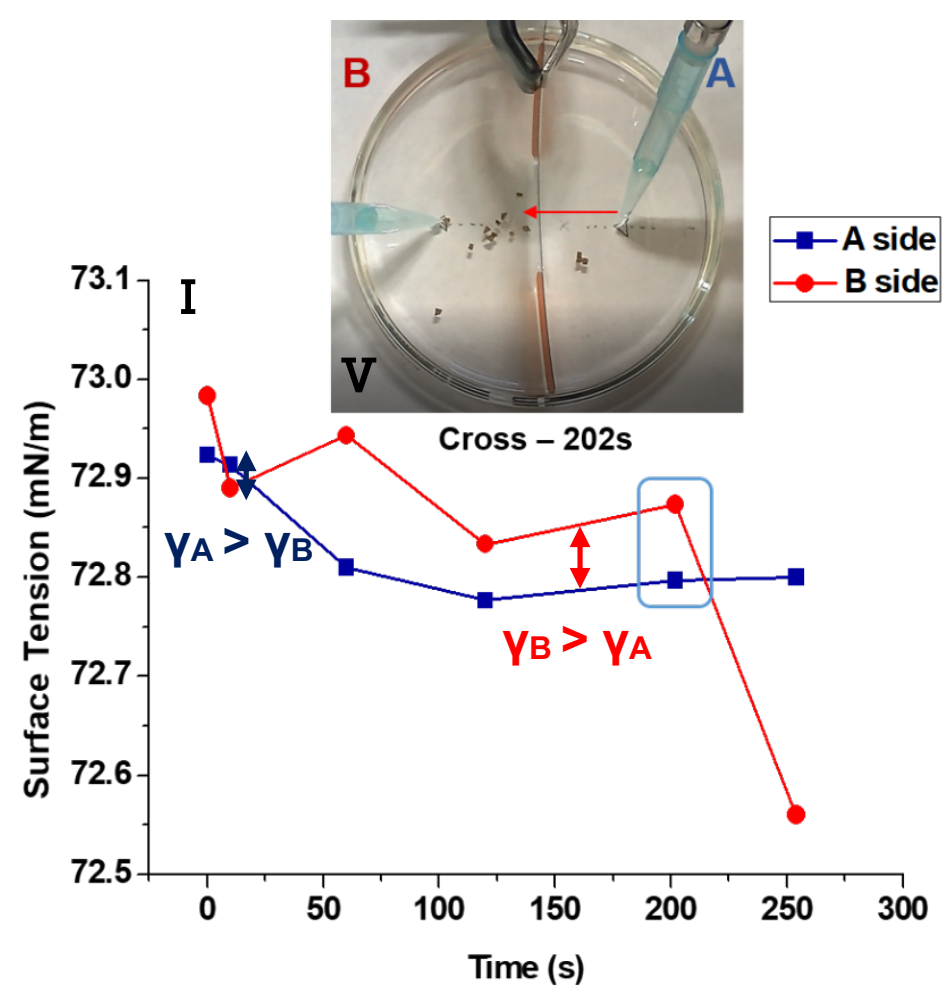

Fig S3. Surface tension vs. time graph of Video S4 and corresponding snapshots. ( I ) Surface tension vs. time graph. 6 sets of 12 samples were taken at $0 \mathrm{~s}$, 10s, 60s, 120s, 202s (crossing), and 254 s (sinking) on both sides. A large surface tension difference between $\mathrm{A}$ and $\mathrm{B}$ sides was shown around the crossing moment $202 \mathrm{~s}$ (picture V) marked by the blue rectangle, indicating the gatecrossing behavior was driven by foraging for surface tension. (II )(VI) Snapshots from the video at sampling times. Red arrow indicated the moving direction of particles. 


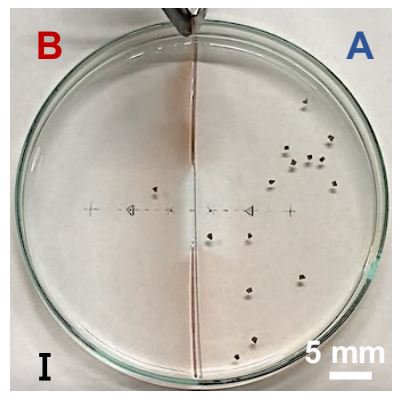

$10 \mathrm{~s}$

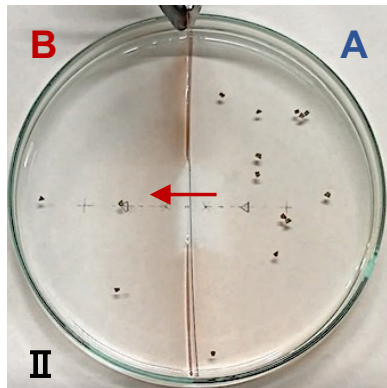

$30 \mathrm{~s}$

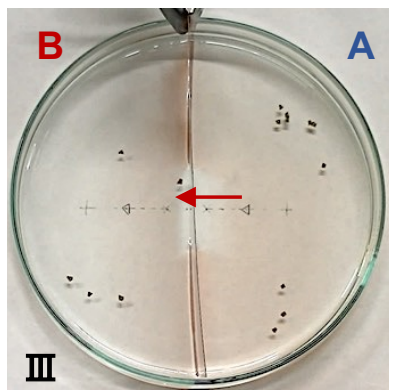

120s

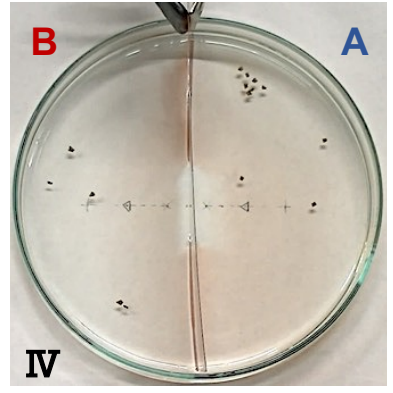

$300 \mathrm{~s}$

Fig S4. Gate-crossing behavior of 15 irregular BQ particles in a large dish $(\mathrm{d}=13.5 \mathrm{~cm})$ at the air-water interface. 5 out of 15 particles crossed the gate from the A to B side, while the flock of 10 particles remained on the A side (IV). Red arrows indicated the moving direction.

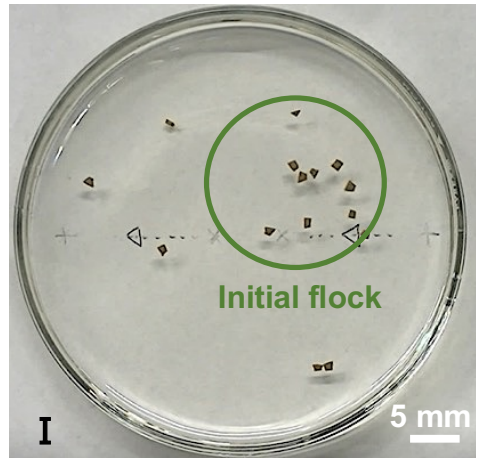

$88 \mathrm{~s}$

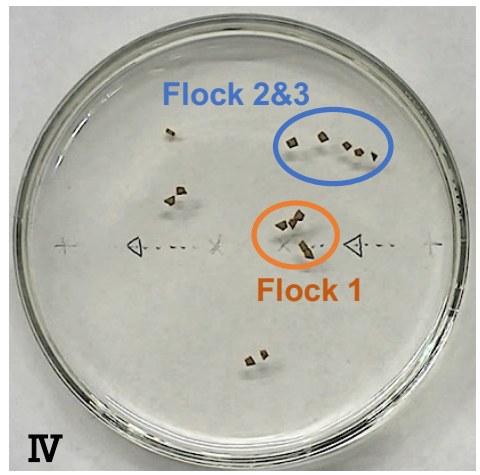

98s

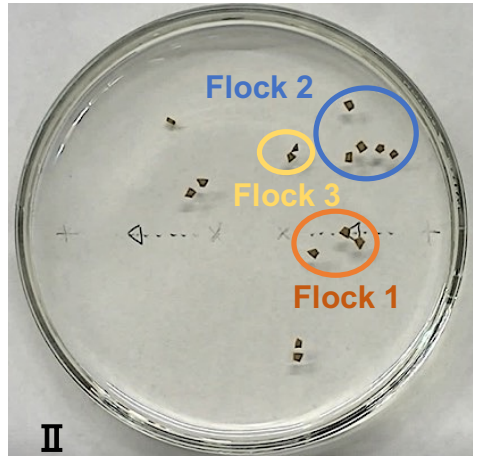

$90 \mathrm{~s}$

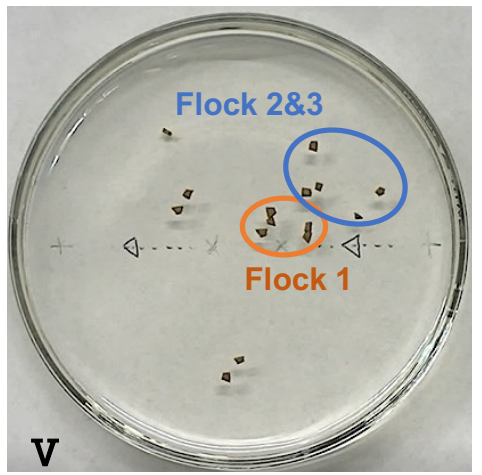

100s

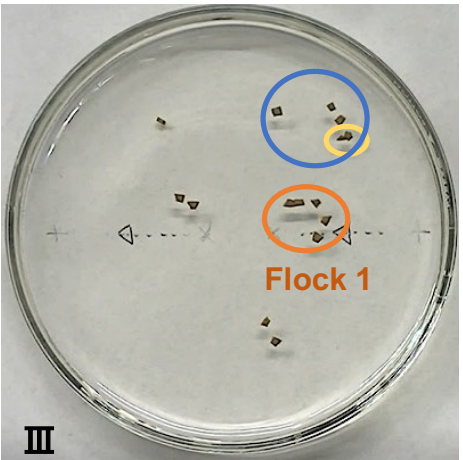

92s

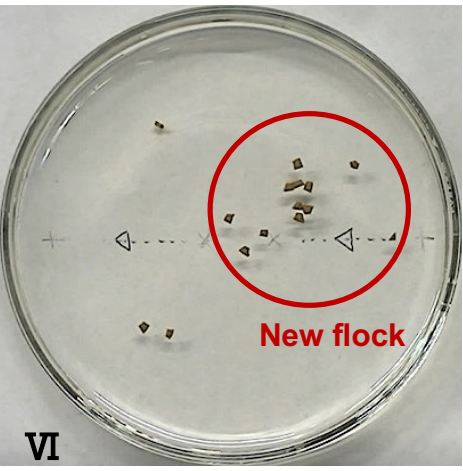

$102 s$

Fig S5. Flocking behavior of 15 irregular BQ particles on pure water surface (no $\mathrm{NaCl}$ ). The initial flock ( I ) broke up into three separate flocks ( II )-(V), and a new flock (VI) was formed later. This flocking behavior is less stable than the case of $\mathrm{NaCl}$ solution. 
BQ particles tend to form separate flocks in pure water instead of one tight flock in $\mathrm{NaCl}$ solution, also the flock breaks up \& reform quickly (Fig S5), making it difficult to control.

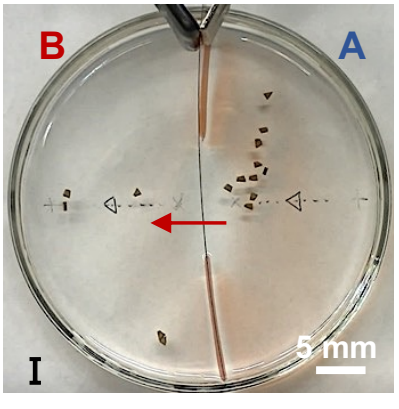

50s

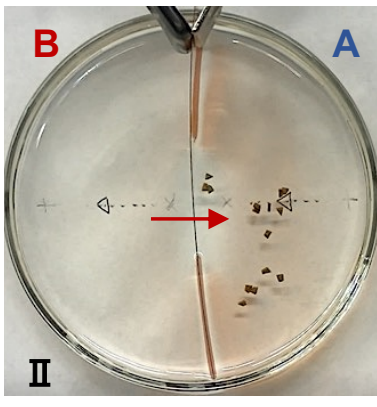

150s

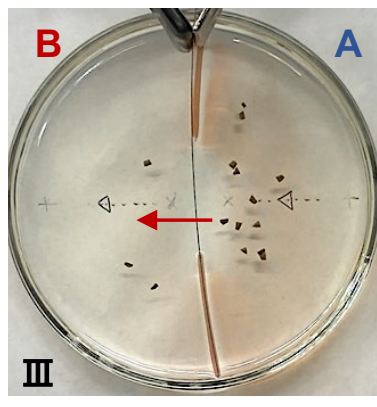

210s

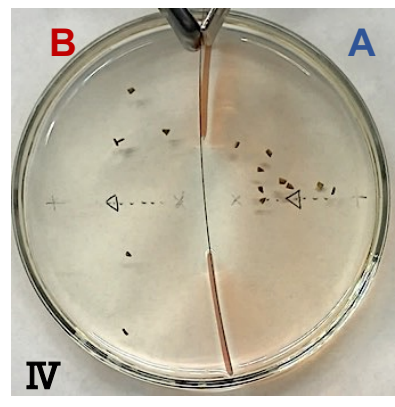

$326 s$

Fig S6. Gate-crossing behavior of 15 irregular BQ particles on pure water surface (no $\mathrm{NaCl}$ ). Particles moved back and forth across the gate frequently ( I )-(III). In the end, 6 out 15 particles crossed the gate to the B side, while the rest 9 remained on the A side (IV). Red arrows indicated the moving direction.

With the gate present, not all BQ particles were observed to cross the gate. Particles appeared to cross the gate and go back more frequently and 'freely' than in $\mathrm{NaCl}$ solution (Fig $\mathrm{S} 6$ ). $\mathrm{NaCl}$ presumably slows down dissolution of $\mathrm{BQ}$ into water and help stabilize the flock. 